\title{
Neglected Isolated Sleeve Fracture of the Superior Pole of the Patella in a Healthy Adult - A Case Report and Review of Literature
}

\author{
Sudhir Shyam Kushwaha ${ }^{1}$, Nitish Kumar ${ }^{1}$, Ajay Bharti ${ }^{1}$, Garima Maurya ${ }^{2}$ \\ Learning Point of the Article: \\ Sleeve fracture of the superior pole of the patella, though extremely rare can present in adults.
}

\section{Abstract}

Introduction: Sleeve fracture (SF) represents an unusual type of patellar fracture and has been reported mainly in children or adolescents. Superior pole fracture in adults is a very rare occurrence because the enthesis of the adult patella is more resilient to avulsion injury as compared to an immature pediatric skeleton. Due to the rarity of these fractures in adults and vague radiographic findings, the chances of missing the fracture are very high.

Case Report: An 18-year-old boy presented to our outpatient department with a history of fall while trying to jump across a wall 6 months ago. At the time of presentation, the patient mainly complained of loss of active extension of the knee in the sitting position and a palpable gap over the upper part of the patella. After radiographic evaluation, a diagnosis of SF was made. The patient was managed surgically, and thereafter, early rehabilitation was done. After 10 weeks post-surgery, the patient was free from any pain, had a full range of motion, and was able to walk without support.

Conclusion: Adults presenting with acute injury to knee with limitation of extension and palpable gap over the superior pole of the patella, a differential diagnosis of SF must be suspected and should be ruled out by clinical examination and relevant investigations.

Keywords: Sleeve fracture, patella, superior pole, adult.

\section{Introduction}

Sleeve fracture (SF) of the patella is described as the traumatic avulsion fracture of the inferior/superior pole, in which a small osseous fragment is pulled, along with a periosteal sleeve and patellar cartilage [1]. SF represents an unusual type of patellar fracture and has been reported mainly in children or adolescents. These fractures account for approximately half of the patellar fractures in children and are common in $8-12$ years of age. Most of these sleeve avulsions involve the inferior patellar pole, whereas the fracture of the superior pole is a rare occurrence [2]. Superior pole fracture in adults is a very rare occurrence because the enthesis of the adult patella is more resilient to avulsion injury as compared to an immature pediatric skeleton. Radiographs may appear normal after the initial trauma. Due to the rarity of these fractures in adults and vague radiographic findings, the chances of missing the fracture are very high [3].

The present case describes the fracture of the superior pole of the patella in an otherwise healthy adult. The patient was initially managed by a local practitioner (quack) and he was not able to identify the fracture. The patient presented to us 6 months after the trauma. Our case highlights that a high degree of suspicion coupled with the clinical examination is required for early diagnosis and good functional outcome of superior pole fractures in the adults.

\section{Case Report}

An 18-year-old boy presented to our outpatient department with a history of fall while trying to jump across a wall 6 months

\begin{tabular}{|c|}
\hline Access this article online \\
\hline $\begin{array}{c}\text { Website: } \\
\text { www.jocr.co.in }\end{array}$ \\
DOI: \\
$10.13107 /$ jocr.2021.v11.i01.1984 \\
\hline
\end{tabular}

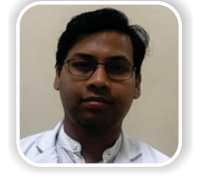

Dr. Sudhir Shyam Kushwah

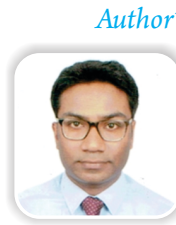

Dr. Nitish Kuma

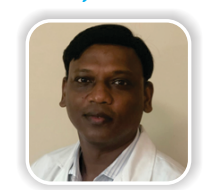

Dr. Ajay Bhart

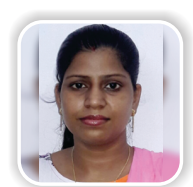

Dr. Garima Maurya
${ }^{1}$ Department of Orthopaedics, All India Institute of Medical Sciences, Gorakhpur, Uttar Pradesh, India, Department of Obstetrics and Gyanecology, BRD Medical College, Gorakhpur, Uttar Pradesh, India.

Address of Correspondence

Dr. Sudhir Shyam Kushwaha,

Department of Orthopaedics, All India Institute of Medical Sciences, Gorakhpur - 273 010, Uttar Pradesh, India.

E-mail: sudhirshyamkushwaha2@gmail.com 


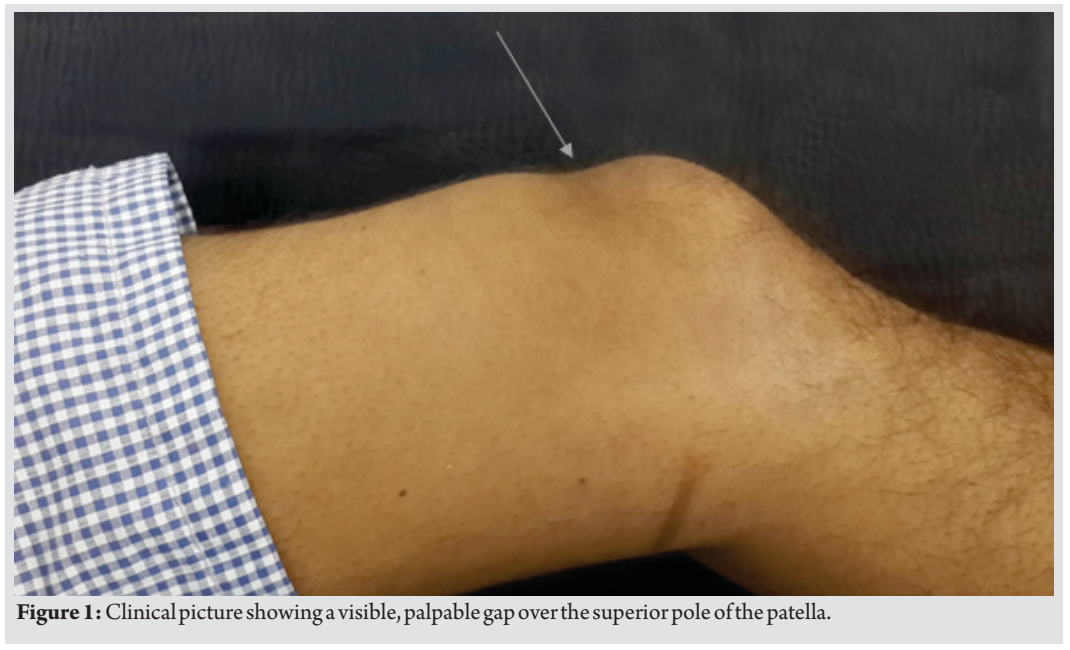

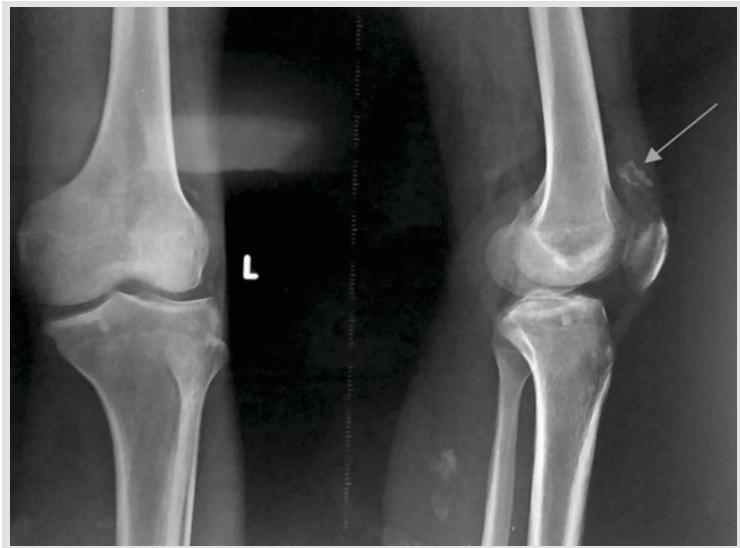

Figure 2: Anteroposterior and lateral view of the knee joint showing sleeve fracture of the superior pole of patella. The arrow represents the sleeve separated from the superior pole. ago. After the injury, the patient was not able to stand and walk. The patient was initially attended by a local practitioner. The initial radiographs were inconclusive for any fracture. The patient was managed by wooden splint and bed rest for 6 weeks were advised. However, after 3 weeks, the patient removed the splint by itself and started walking with a wooden stick.

At the time of presentation to us, the patient was able to walk normally. The patient mainly complained of loss of active extension of the knee in the sitting position and a palpable gap over the upper part of the patella (Fig. 1). The patient was able to actively flex his knee, but an active extension was minimally possible. The patient was able to extend his limb by internal rotation most probably using tensor fascia lata.

Concerning the extent of the injury, the patient underwent radiographic evaluation which showed a small bony avulsion at the upper pole of the patella. Anteroposterior and lateral radiograph (Fig. 2) were taken, which showed the fracture line along with displaced fragments, which appeared to be a sleevelike fracture of the upper pole of the patella.

After the complete evaluation, the patient was operated under spinal-epidural anesthesia. The fracture was approached through a suprapatellar incision. We found that the articular cartilage of the patella and portion of patellar retinaculum was avulsed, along with small bony fragments. The fragment was anatomically reduced and fixed with 4 non-resorbable osseous sutures (Ethibond No. 5). Subsequently, to provide more stability, a cerclage wiring was also done (Fig. 3).

Post-operatively, the knee was kept in extension with a longknee brace from groin to ankle for 3 weeks duration. On the 11th-day post-surgery, sutures were removed and a long knee brace was continued. After 3 weeks, the knee brace was removed, and active continuous passive motion assisted exercises were started. As the pain subsided isometric quadriceps, exercise was started. After 10 weeks post-surgery, the patient was free from any pain, had a full range of motion, and was able to walk without support.

\section{Discussion}

The present case report highlights the importance of early diagnosis and treatment of SF of the patella in adults. A plain radiograph may be misleading, and the presence of a thin osteochondral fragment may be missed due to the rarity of these fractures in adults. In the present case, the fracture was neglected for 6 months. Nonetheless, surgical treatment and early rehabilitation after the treatment can yield good results.

The term SF was first described by Houghton et al (1979), as a sleeve of the cartilage pulled off the main bony patella [4]. They reported three cases of SF of the inferior pole of the patella. Since then, several authors have reported SF of the inferior pole of the patella in children, but reports of the similar fracture at the superior pole are rare and among the adult population only a few cases have been reported. As far as English literature is concerned, we are reporting the 6 th case of the SF of the patella in adults and probably the 1 st neglected case of the superior pole

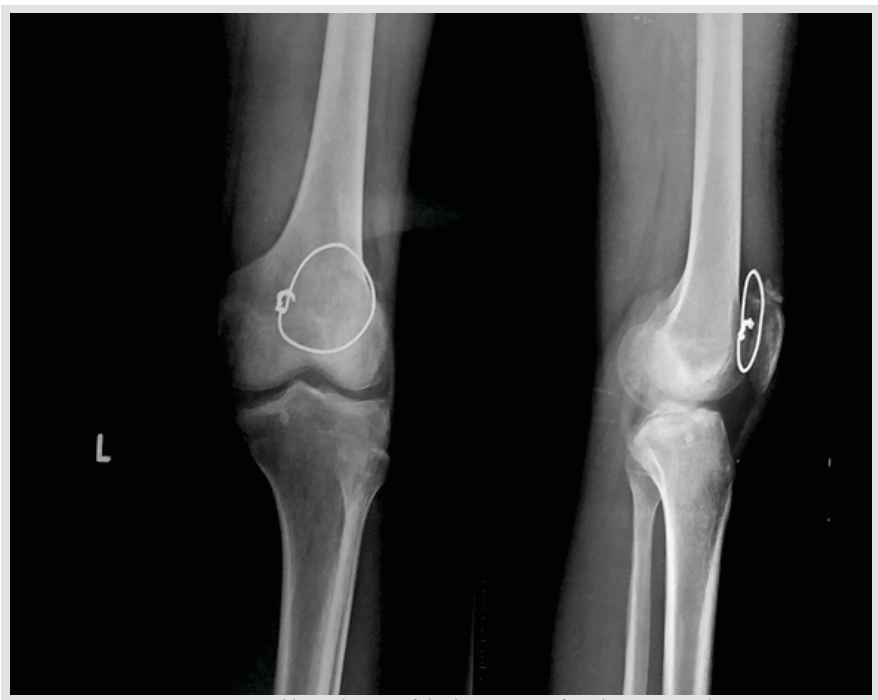

Figure 3: Anteroposterior and lateral view of the knee joint after the surgery showing reposition of the sleeve to the superior pole and re-enforcement by cerclage wiring. 
Table 1: Sleeve fracture of superior pole of patella in adults reported in the English literature

\begin{tabular}{|c|c|c|c|c|c|c|c|}
\hline Case & Authors & Year & Age/sex & Mechanism of injury & Treatment & Follow-up & Associated lesion \\
\hline Case 1 & Ro et al. [3] & 2014 & $24 \mathrm{y} / \mathrm{M}$ & $\begin{array}{c}\text { Forceful passive } \\
\text { physiotherapy following cast } \\
\text { immobilization }\end{array}$ & Surgery & $\begin{array}{l}26 \text { months, return to sports } \\
\text { activity }\end{array}$ & $\begin{array}{c}\text { Posterior cruciate ligament } \\
\text { tear, posterolateral corner } \\
\text { injury }\end{array}$ \\
\hline Case 2 & $\begin{array}{c}\text { Teixeiraet al . } \\
{[5]}\end{array}$ & 2013 & $22 \mathrm{y} / \mathrm{M}$ & $\begin{array}{l}\text { Forceful kick to ball during } \\
\text { the soccer match }\end{array}$ & Conservative & $\begin{array}{c}3 \text { weeks, normal physical } \\
\text { activity }\end{array}$ & Healthy \\
\hline Case 4 & Xie et al. [2] & 2014 & $19 \mathrm{y} / \mathrm{F}$ & Fall while jumping & Surgery & Full recovery after 6 months & Healthy \\
\hline Case 5 & $\begin{array}{c}\text { Hemeleers and } \\
\text { Siemons [7] }\end{array}$ & 2019 & $19 \mathrm{y} / \mathrm{M}$ & Fall on ground & Conservative & Follow-up not reported & Healthy \\
\hline
\end{tabular}

of patella among the healthy adults. The clinical data of the previously reported cases are summarized in Table $1[2,3,5,6,7]$.

In our case, the patient was a case of neglected SF of the superior pole. There is no data available over the management of the neglected SF.

SF is mostly limited to children and adolescents with the most common presentation in the $8-12$ years of age group [ 8 ]. These fractures account for up to $57 \%$ of all patella fractures under 16 years of age [2]. This age range predominance can be explained as a more flexible joint capsule, articular cartilage, and more flexible ligaments in the children's results in joint laxity and acts as a protective mechanism of patella from traumatic injury. A similar mechanism stood behind the more common avulsion injury of cartilage or bone than the rupture of the tendon. Further, the osteochondral junction in the immature skeleton of the children is more vulnerable to traumatic injury than the enthesis of a fully ossified patella in adults [3].

Patella is the largest sesamoid bone in the body which develops in the tendon of the quadriceps femoris. Ossification centers appear at 2-3 years, but it may be delayed if 6 years and fuses by the age of 16-18 years. Among these centers the ossification center of the superior and inferior poles are predominantly cartilaginous during childhood and early adolescence. Due to rapid growth with osteochondral activity at the periphery of the patella and high intensity of the sports in the adolescence, they are more susceptible to these types of fractures [9].

$\mathrm{SF}$ is different from simple avulsion due to the periosteal sleeve attached to the fracture fragment. The sleeve, thus, had an osteogenic tissue and if not treated timely it will continue to form new bone. Hence, the missed diagnosis of the SF or delay in treatment leads to new bone formation and results in permanent disability including quadriceps weakness, extensor lag, patella Magna, and patellofemoral arthrosis. Therefore, early diagnosis and treatment are necessary for a good prognosis [10].

SF in children usually involves the inferior pole, and superior pole involvement is rare. However, among the adult's superior pole fractures are more common. The mechanism of fracture is different in these two types. It is proposed that $\mathrm{SF}$ of the inferior pole is due to a rapid contraction of the quadriceps muscle as occurs in jumping and skateboarding. In contrast, superior pole fracture occurs as a result of rapid quadriceps muscle contraction with knee flexion at the time of injury resulting in more force transmitted to the upper pole as compared to the inferior pole $[5,11]$.

The previous reports have emphasized that one may miss the diagnosis unless there is a high index of suspicion. Acute cases usually present with a history of a painful and swollen knee, inability to extend/flex the knee actively, and negative straight leg raise test. A history of rapid muscle contraction with forced flexion during the injury may suggest a superior pole fracture. A plain radiograph may include one or more features: Joint effusion, soft-tissue swelling, a thin shell of the bone, and an anterior tilt of patella. However, these features are not specific for the superior pole fracture [12].

Additional diagnostic modalities such as ultrasound (USG) and magnetic resonance imaging can help in the diagnosis of the fracture and can quantify the extent of the fracture also. However, the use of these modalities is recommended if the diagnosis is doubtful after clinical examination and plain radiographs [11]. In the present case, a palpable gap due to retraction of the ligament, loss of extension, and a sleeve of the cortical bone suggested the diagnosis. HRUSG of the knee further differentiated the quadriceps tear from the SF.

SF can be managed both surgically and conservatively $[13,14]$. Conservative management is usually indicated in minimally 
displaced fractures, but the results are not very satisfying. Further, conservative management can result in retraction of the quadriceps muscle and joint stiffness. Therefore, surgical management of the superior pole is preferred over the conservative treatment. Surgical management includes anatomical reduction and fixation of the sleeve to the patella. Fixation can be done by transosseous sutures, ethibond, intraosseous anchors, or tension band wiring. Post-surgery early rehabilitation is needed to yield good functional results and range of motion [15].

\section{Conclusion}

SF of the patella usually occurs in children and preferably involves the inferior pole, but they can also occur in adults and involve the superior pole also. As the avulsed fragment primarily contains cartilaginous tissue, a plain radiograph may be misleading. Late recognition, leading to neglected fracture, can occur; therefore, adults presenting with acute injury to knee with limitation of extension and palpable gap over the superior pole of the patella, a differential diagnosis of SF must be suspected and should be ruled out by clinical examination and relevant investigations.

\section{Clinical Message}

SF of the patella in adults is prone to be missed since the radiological features may be inconclusive for the fracture. Nonetheless, a neglected SF should be managed by open reduction and fixation of the osseous fragment to the patella followed by early rehabilitation to get a good functional result.

\section{References}

1. Schmidt-Hebbel A, Eggers F, Schütte V, Achtnich A, Imhoff AB. Patellar sleeve avulsion fracture in a patient with Sinding-Larsen-Johansson syndrome: A case report. BMC Musculoskelet Disord 2020;21:267.

2. Xie L, Xu H, Zhang L, Xu R, Guo Y. Sleeve fracture of the adult patella: Case report and review of the literature. Medicine (Baltimore) 2017;96:e7096.

3. Ro KH, ParkJH, Kim MJ, Lee DH. Rare sleeve fracture of the superior patella pole in an adult due to forceful passive physiotherapy following cast immobilization. Knee 2014;21:600-4.

4. Houghton GR, Ackroyd CE. Sleeve fractures of the patella in children: A report of three cases. J Bone Joint Surg Br 1979;61:165-8.

5. Teixeira PA, Lecocq S, Moisei A, Chanson A, Louis M, Blum A. Sleeve-like avulsion fracture of the superior pole of the patella in a healthy adult. Diagn Interv Imaging 2013;94:108-11.

6. Kakazu T, Tatemoto H, Kawamura M, Sugita T. Sleeve fracture of the upper pole of the patella in an adult with osteogenesis imperfecta. Injury 2003;34:793-4.

7. Hemeleers W, Siemons W. Superior sleeve avulsion of the patella.J Belg Soc Radiol 2018;102:65.

Conflict of Interest: Nil

Source of Support: Nil

Consent: The authors confirm that informed consent was obtained

from the patient for publication of this case report
8. Lin SY, Lin WC, Wang JW. Inferior sleeve fracture of the patella.J Chin Med Assoc 2011;74:98-101.

9. Hunt DM, Somashekar N. A review of sleeve fractures of the patella in children. Knee 2005;12:3-7.

10. Van Isacker T, De Boeck $H$. Sleeve fracture of the upper pole of the patella: A case report. Acta Orthop Belg 2007;73:114-7.

11. Kumar K, Knight DJ. Sleeve fracture of the superior pole of patella: A case report. Knee Surg Sports Traumatol Arthrosc 2005;13:299-301.

12. Klerx-Melis F, Watt I. The mechanism and diagnosis of a sleeve fracture of the upper pole of the patella in children. EurJ Radiol Extra 2006;59:67-70.

13. Sousa PL, Stuart MJ, Prince MR, Dahm DL. Nonoperative Management of Minimally Displaced Patellar Sleeve Fractures. J Knee Surg. 2019 Aug 21. doi: 10.1055/s-00391694742. Epub ahead of print.

14. Gettys FK, Morgan RJ, Fleischli JE. Superior pole sleeve fracture of the patella: A case report and review of the literature. Am J Sports Med 2010;38:2331-6.

15. Tsubosaka M, Makino T, Kishimoto SI, Yamaura K. A case report of sleeve fracture of the patella in a shield. J Orthop Case Rep 2016;6:24-7.

\section{How to Cite this Article}

Kushwaha SS, Kumar N, Bharti A, Maurya G. Neglected Isolated Sleeve Fracture of the Superior Pole of the Patella in a Healthy Adult - A Case Report and Review of Literature. Journal of Orthopaedic Case Reports 2021 January; 11(1): 104-107. 